\title{
A Novel Intracellular Target for Anti-Allergic Medications
}

S. Rastogi**, A. Calabro*, C. Queenan*, D. Becker*, D. Leonardi**

* Bergen County Academies, Nano-Structural Imaging Lab, 200 Hackensack Avenue, Hackensack, NJ 07601

** Bergen County Academies, Biotechnology Lab, 200 Hackensack Avenue, Hackensack, NJ 07601

Curcumin, a potent spice found in Asian cuisines, has been found to have anti-allergic properties, however; the mechanism of action for these properties has yet to be fully elucidated. Allergic reactions are caused by the release of histamine through the degranulation of mast cells. Degranulation is, in part, from the translocation of polymerized microtubules in a calciumindependent fashion and F-actin ring disassembly in a calcium-dependent fashion; both of which are mediated by the protein RhoA [1].

This study demonstrates that treating rat basophilic leukemia cells (RBL-2H3) with curcumin $(1 \mu \mathrm{g} / \mathrm{ml})$ and stimulating them to degranulate significantly decreases the release of histamine $(p<0.05)$ and decreases the activation of RhoA $(p<0.05)$. Cells were treated with either curcumin; anti-rat immunoglobulin $\mathrm{E}$ (IgE) (which stimulates degranulation and the release of histamine); or both curcumin and $\operatorname{IgE}$.

To determine if curcumin affects microtubule translocation and/or F-actin ring disassembly, its effect on microtubules, F-actin rings and calcium levels were analyzed by fluorescent microscopy using Paclitaxel, phalloidin, and indo-1AM dye, respectively. In degranulating cells, the microtubules were located around the periphery due to the translocation of vesicles to the outer membrane for the release of histamine (Figure 1A). When degranulating cells were treated with curcumin, bright microtubule aggregations formed in the middle of the cells (Figure 1B). Curcumin caused microtubule aggregation $(p<0.05)$, increased disassembly of $F$-actin rings $(p<0.05)$, but had no effect on intracellular calcium levels. This suggests that curcumin inhibits degranulation by impeding functional microtubule polymerization and translocation.

To discern any morphological alterations to mast cells due to the curcumin treatment, cells were prepared for imaging by transmission electron microscopy (TEM). Cells were fixed in 4\% glutaraldehyde $/ 2 \%$ formaldehyde in $0.2 \mathrm{M}$ sodium cacodylate buffer, $\mathrm{pH} 7.4$, scraped from the wells with Teflon ${ }^{\circledR}$ and gently spun down to form a pellet. The pellet was post-fixed in $2 \%$ osmium tetroxide, dehydrated in a graded series of acetone, infiltrated and cured in epoxy resin. 100nm sections were collected onto 200 mesh copper grids and post-stained with $4 \%$ uranyl acetate and $0.5 \%$ lead citrate.

Untreated control cells and cells treated with curcumin both appeared compact and well-defined, indicating that curcumin itself was not causing visible damage to the cells (Figures 2A\&B). However, in cells treated with $\mathrm{IgE}$, the cell membranes appeared discontinuous, most likely because of the large amount of exocytosis occurring due to degranulation (Figure 2C). The cells treated with both IgE and curcumin had morphologies more similar to the control than the IgE treated cells (Figure 2D). The TEM images show that curcumin inhibits the release of histamine in these cells by the presence of multiple granules and the lack of apparent exocytosis. Furthermore, the data 
provided in this study shows that curcumin could prove to be useful as an allergic medication, and that microtubules may be a novel intracellular target for anti-allergic medications. Future experiments would involve immunogold labeling of curcumin and histamine for TEM visualization to more accurately determine curcumin's location within the cell.

\section{References}

[1] K. Nishida, et al., The Journal of Cell Biology. 170 (2005) 115-126.

[2] The authors would like to acknowledge the administration of the Bergen County Technical Schools for their continued support of the research program.
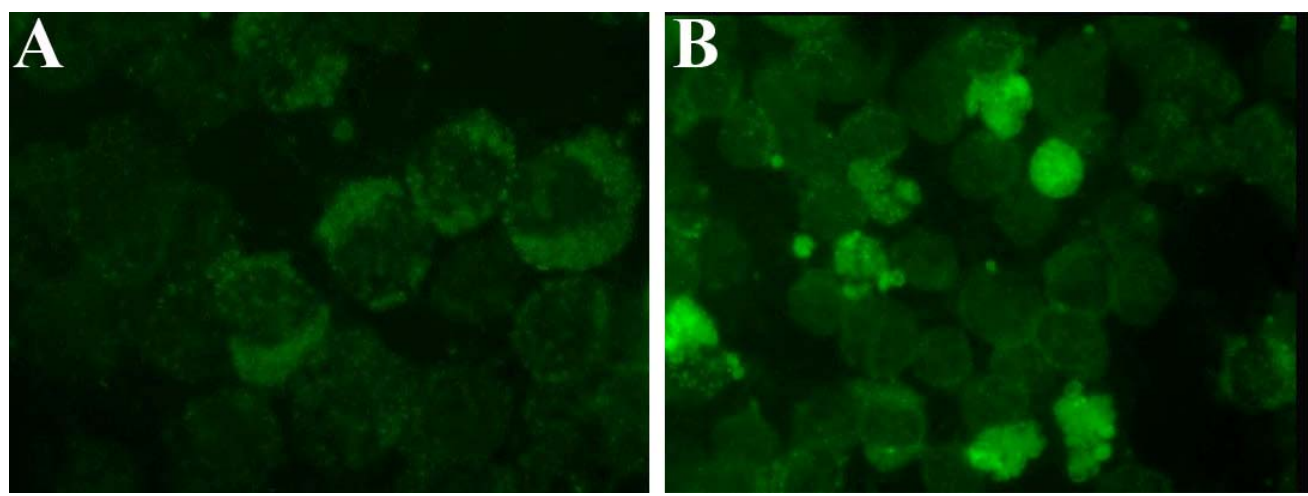

Figure 1: Fluorescent microscopy of treated RBL-2H3 cells stained with $1 \mu \mathrm{M}$ Paclitaxel Oregon Green 488 conjugate (Invitrogen). (A) Degranulating cells with microtubules located around the periphery. (B) Degranulating cells treated with curcumin show bright microtubule aggregations.
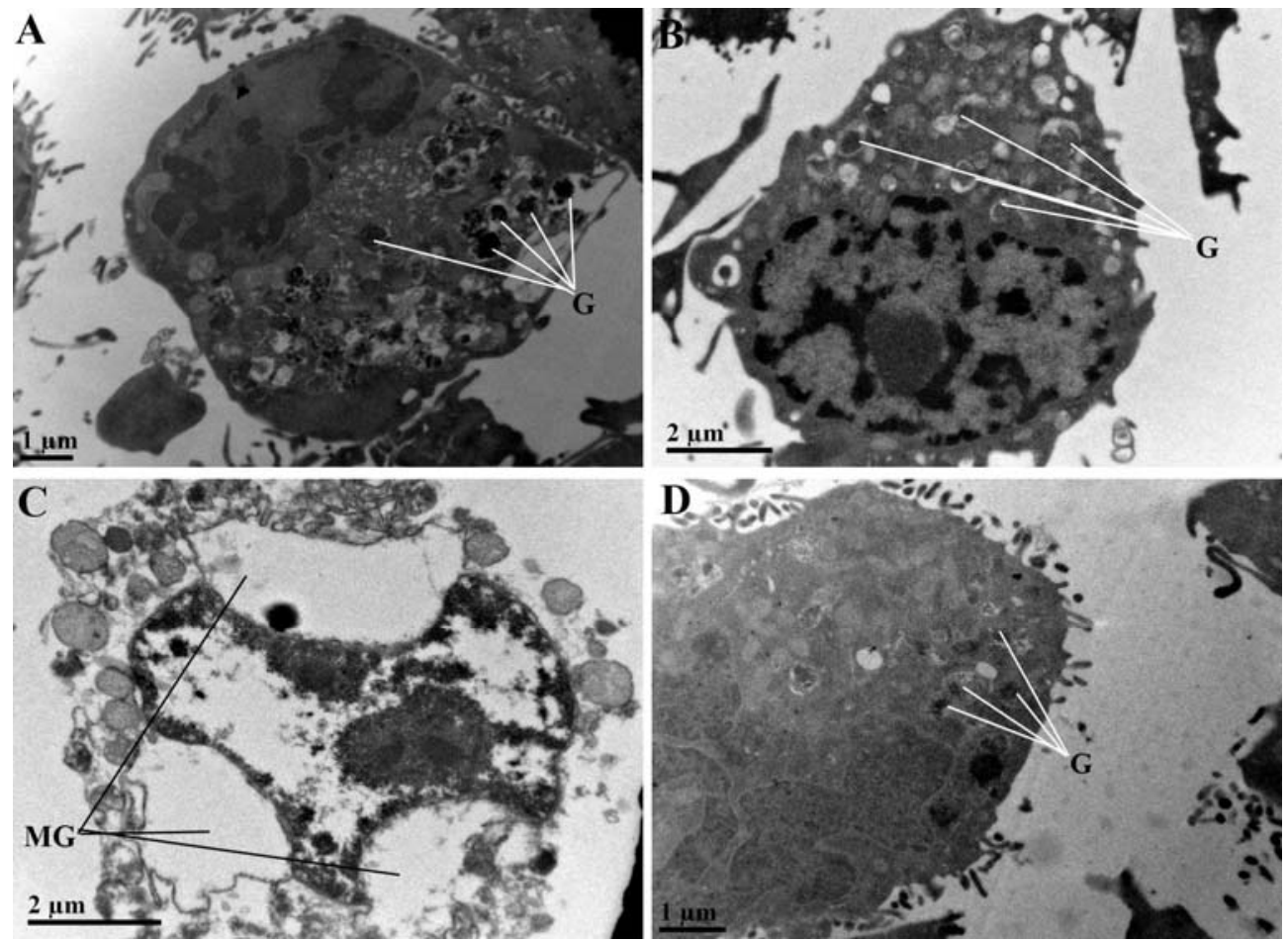

Figure 2: TEM images of treated RBL-2H3 cells. (A) Untreated control cells. (B) Cells treated with curcumin $(1 \mu \mathrm{g} / \mathrm{mL})$. (C) Cells stimulated to degranulate with IgE. (D) Cells treated with curcumin and stimulated to degranulate with IgE. G denotes immature granules with histamine inside. MG represents mature granules where histamine has been released. 